Fehlheilungen

Maximilian Faschingbauer · Olaf Pingen · Sarah Strametz · Michael Wenzl · Christian Jürgens

Abteilung für Unfall- und Wiederherstellungschirurgie, BG-Unfallkrankenhaus Hamburg

\title{
Rekonstruktion und Stabilisierung nach fehlverheilten Frakturen und Pseudarthrosen im Femurbereich mit winkelstabilen Implantaten
}

\section{Zusammenfassung}

Für das Femur stehen zahlreiche Implantate für eine Osteosynthese zur Verfügung. Das Implantat der Wahl für die Frakturversorgung ist sicher der Marknagel, sei er aufgebohrt oder unaufgebohrt. Beim Auftreten von Komplikationen, wie z. B. Fehlstellungen oder ausbleibender knöcherner Durchbauung, gewinnen andere Implantate wieder an Bedeutung. Insbesondere nach mehrfachen Voroperationen erweisen sich multidirektional winkelstabile Fixateur-interne-Systeme als hilfreich, um eine sichere knöcherne Konsolidierung zu erreichen. Wir berichten über 64 Patienten, die mit einem Fixateur-interne-System bei Fehlstellungen und Pseudarthrosen von 1993-2001 operativ versorgt wurden. Bei allen 59 nachuntersuchten $\mathrm{Pa}$ tienten kam es zu einem knöchernen Durchbau der Korrekturosteotomien und Pseudarthrosen. Trotz des hohen Anteils an intraoperativ positiven Keimnachweisen von $18,6 \%$ trat keine Infektion auf.

\section{Schlüsselwörter}

Pseudarthrosen · Fehlstellungen · Korrekturosteotomien - Fixateur-interne-Systeme . Multidirektionale Winkelstabilität
Bei Pseudarthrosen und Fehlstellungen im Femurbereich handelt es sich meist um Komplikationen der operativen Frakturversorgung. Die Inzidenz für postoperative Fehlstellungen, insbesondere Rotationsfehlstellungen, wurde zwischen 1 und 28\% [13, 14] angegeben. Nach intramedullärer Nagelung von Oberschenkelschaftfrakturen wurden Pseudarthrosenraten zwischen 1 und $25 \%[1,2,3,5,10,17,18]$ beschrieben. Der vermehrte Einsatz von soliden, unaufgebohrten Nägeln führte zu längeren Durchbauungszeiten und steigenden Pseudarthroseraten $[4,8,9]$.

Insbesondere bei Problempatienten mit mehrfachen Voroperationen, schlechten Durchblutungs- und Weichteilverhältnissen, ungenügender Knochenqualität und Osteoporose ist ein Implantat gefordert, das eine hohe Stabilität aufweist und der geschädigten Durchblutung der mehrfach voroperierten Region Rechnung trägt. Wir setzen hierfür multidirektional winkelstabile Implantate, den Druckplattenfixateur interne und den Kondylen-TiFix, ein $[7,19]$.

\section{Material und Methode}

Der Druckplattenfixateur für den Oberschenkel (Litos, Hamburg) besteht aus Reintitan und liegt in 2 Längen ( $234 \mathrm{~mm}$ und $250 \mathrm{~mm}$ ) vor (Abb. 1). Er ist nach dem Low-contact-Prinzip geformt und weist in der Mitte eine Welle auf. Die
Schrauben können mit einem Winkel bis $\mathrm{zu} 40^{\circ}$ zur Plattenachse eingebracht werden. Die Winkelstabilität wird durch das Aufschrauben von Druckplatten, welche die Schraubenköpfe verklemmen, erreicht. Einsatzgebiet sind v. a. Versorgungen im Schaftbereich (Abb. 1).

Für den metaphysären Bereich liegt ein multidirektional winkelstabiles Titanfixateur System (Kondylen-TiFix, Litos, Hamburg) vor (Abb. 2). Die Platte besteht aus einem weicheren Titan (Titan o) als die hochfeste Reintitanschraube (Ti$\tan$ 4). Der Schraubenkopf trägt ein Gewinde mit einer unterschiedlichen $\mathrm{Ge}$ windesteigung, sodass es zu einem Anpressen der Platte an die Knochenoberfläche kommt. Aufgrund der Plattendicke ist es notwendig, das Gewinde im Schraubenloch vorzudrängen, um den Kraftaufwand für das Einbringen des Schraubenkopfs in die Platte zu reduzieren. Die Kondylenplatte steht in 4 verschiedenen Längen $(165 \mathrm{~mm}, 180 \mathrm{~mm}, 200 \mathrm{~mm}, 215$ $\mathrm{mm}$ ), jeweils für die rechte und die linke Seite, zur Verfügung. Auch hier können die Schrauben in einem Winkel bis zu $30^{\circ}$ frei eingebracht werden.
Abb. 1 Druckplattenfixateur interne für das Femur

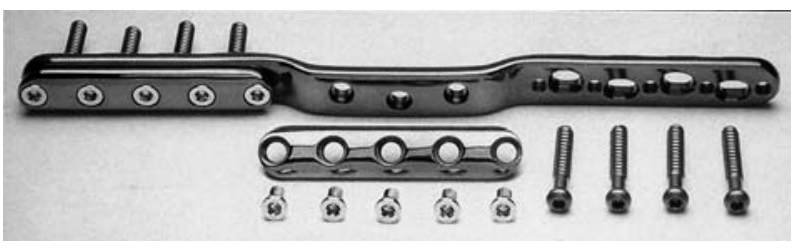


M. Faschingbauer $\cdot 0$. Pingen $\cdot$ S. Strametz . M.Wenzl $\cdot$ C. Jürgens

\section{Reconstruction and stabilization of malalignment and pseudarthroses of the femur with internal fixator systems with locked screws}

\section{Abstract}

Numerous implants are available for internal fixation of fractures of the femur. Preference is generally given to unreamed or reamed intramedullary nails. In the case of complications, such as a malalignment or nonunion, other implants have advantages. Internal fixation systems with multidirectional angular stability have proved especially helpful in the achievement of complete osseus consolidation in patients who have already undergone several operations. We report on $64 \mathrm{pa}-$ tients who were treated operatively with internal fixator systems from 1993 to 2001 after developing malalignment or pseudarthrosis. In all 59 patients in whom followup examinations were possible, complete osseus consolidation was recorded. Although intraoperative microbiological cultures were positive in $18.6 \%$ of our patients, no cases of infection were observed.

\section{Keywords}

Pseudarthrosis $\cdot$ Malalignment $\cdot$ Correctional osteotomy $\cdot$ Internal fixator systems $\cdot$ Plates with locked screws
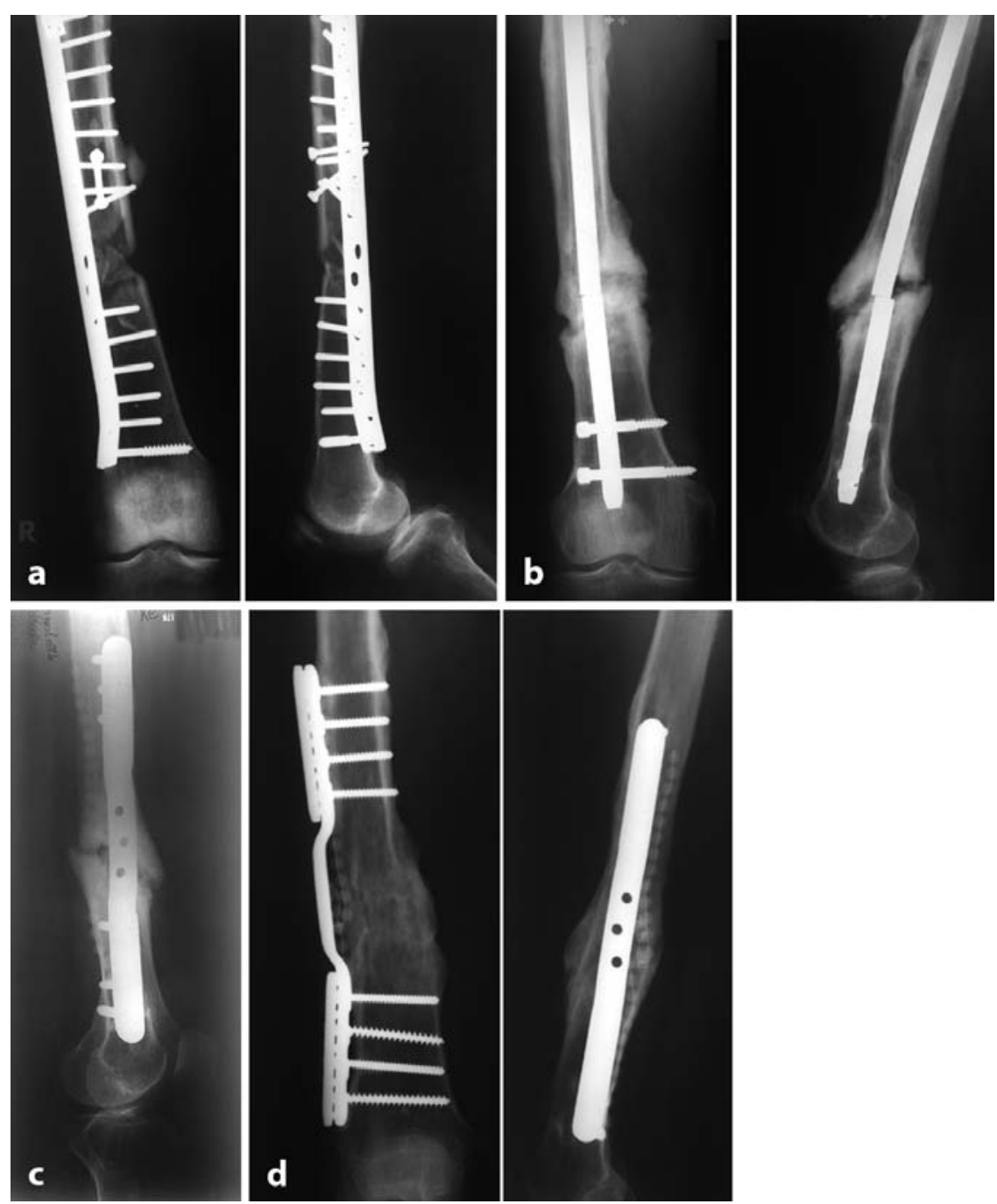

Abb. 3 a-d $\Delta$ 61-jähriger Patient mit Femurschaftfraktur rechts, a Versorgung mit langer LCDC-Platte, $b$ bei ausbleibender knöcherner Durchbauung erfolgte der Verfahrenswechsel mit Umstieg auf Marknagel, Bruch des Marknagels bei Pseudarthrosenbildung, $c$ Entfernung des Marknagels und Stabilisierung mit Druckplattenfixateur und Anlagerung von Spongiosa und PMMA-Ketten, $d$ knöcherner Durchbau bei liegendem Osteosynthesematerial

Beide Platten können mit herkömmlichen Biegeinstrumenten geschränkt und der Anatomie des Kno-

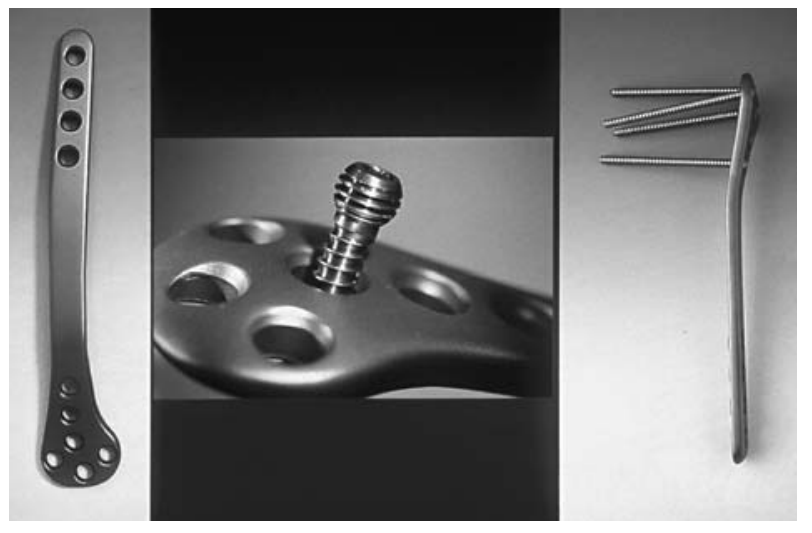

Abb. $2<$ TiFix-Kondylenplatte für das Femur. Die Schrauben können - bei erhaltener Winkelstabilität bis zu $30^{\circ}$ divergierend eingebracht werden chens angepasst werden, ohne an Winkelstabilität zu verlieren (Abb. 2).

Im BG-Unfallkrankenhaus Hamburg wurden von 1993-200164 Patienten mit Fixateur-interne-Systemen im Femurbereich operativ versorgt. Dabei handelte es sich um 24 Fehlstellungen und 40 Pseudarthrosen. Bei den Pseudarthrosen überwogen die atrophen Pseudarthrosen $(n=31)$. Das Durchschnittsalter betrug 42,6 Jahre (11-78 Jahre). Eine primäre Spongiosaplastik wurde 58-mal vorgenommen, PMMA-Ketten wurden 59-mal eingelegt. Intraoperativ wurden regelmäßig Abstriche zur bakteriologischen Untersuchung entnommen.

Im Durchschnitt waren bei den Fehlstellungen 2,6 Voroperationen (1-4), 
bei den Pseudarthrosen 4,3 Voroperationen (1-9) durchgeführt worden.

\section{Ergebnisse}

Es konnten 59 Patienten nachuntersucht werden, 5 Patienten waren unbekannt verzogen.

Bei allen Patienten kam es zu einer knöchernen Konsolidierung der Korrekturosteotomien bzw. Pseudarthrosen. Alle Patienten belasten voll. Bei den intraoperativ gewonnenen Abstrichen war der Keimnachweis in $18,6 \%$ positiv. Es handelte sich fast ausschließlich um Staphylococcus epidermidis, der v. a. bei den atrophen Pseudarthrosen nachweisbar war. Ein klinisch manifester Infekt trat nicht auf. Bei 2 Patienten wurde aufgrund verzögerter knöcherner Durchbauung der Pseudarthrose eine sekundäre Spongiosaplastik durchgeführt. In beiden Fällen kam es zu einem knöchernen Durchbau.

\section{Komplikationen}

Ein Plattenbruch war 3-mal zu verzeichnen.

Beim 1. Patienten kam es nach plattenosteosynthetischer Versorgung einer supra- und diakondylären Oberschenkelfraktur mit einer breiten LCDC-Platte nach 5 Monaten zu einem Plattenausriss. Es wurde eine Reosteosynthese mit einem Kondylen-TiFix durchgeführt. Nach weiteren 5 Monaten kam es zu einem Plattenbruch des Kondylen-TiFix, weshalb eine Reosteosynthese mit Druckplattenfixateur und zusätzlicher Spongiosaplastik vorgenommen wurde. Bei diesem Patienten lag eine chronischlymphatische Leukämie vor, welche zytostatisch therapiert wurde.

Beim 2. Patienten wurde nach einer beruhigten Femurosteitis eine Pseudarthrose mittels Druckplattenfixateur stabilisiert. Hier kam es zu einem Plattenbruch, weshalb ein erneuter Druckplattenfixateur mit Spongiosaplastik eingebracht wurde.

Beim 3. Patienten handelte es sich um einen uneinsichtigen, übergewichtigen Patienten, der aufgrund einer Femurpseudarthrose mit einem Druckplattenfixateur versorgt worden war. Aufgrund von Beschwerdefreiheit belastete der Patient voll. Auch hier kam es zu einem Plattenbruch. Bei diesem Patienten war der intraoperative Keimabstrich positiv. Es erfolgte die erneute Versor-
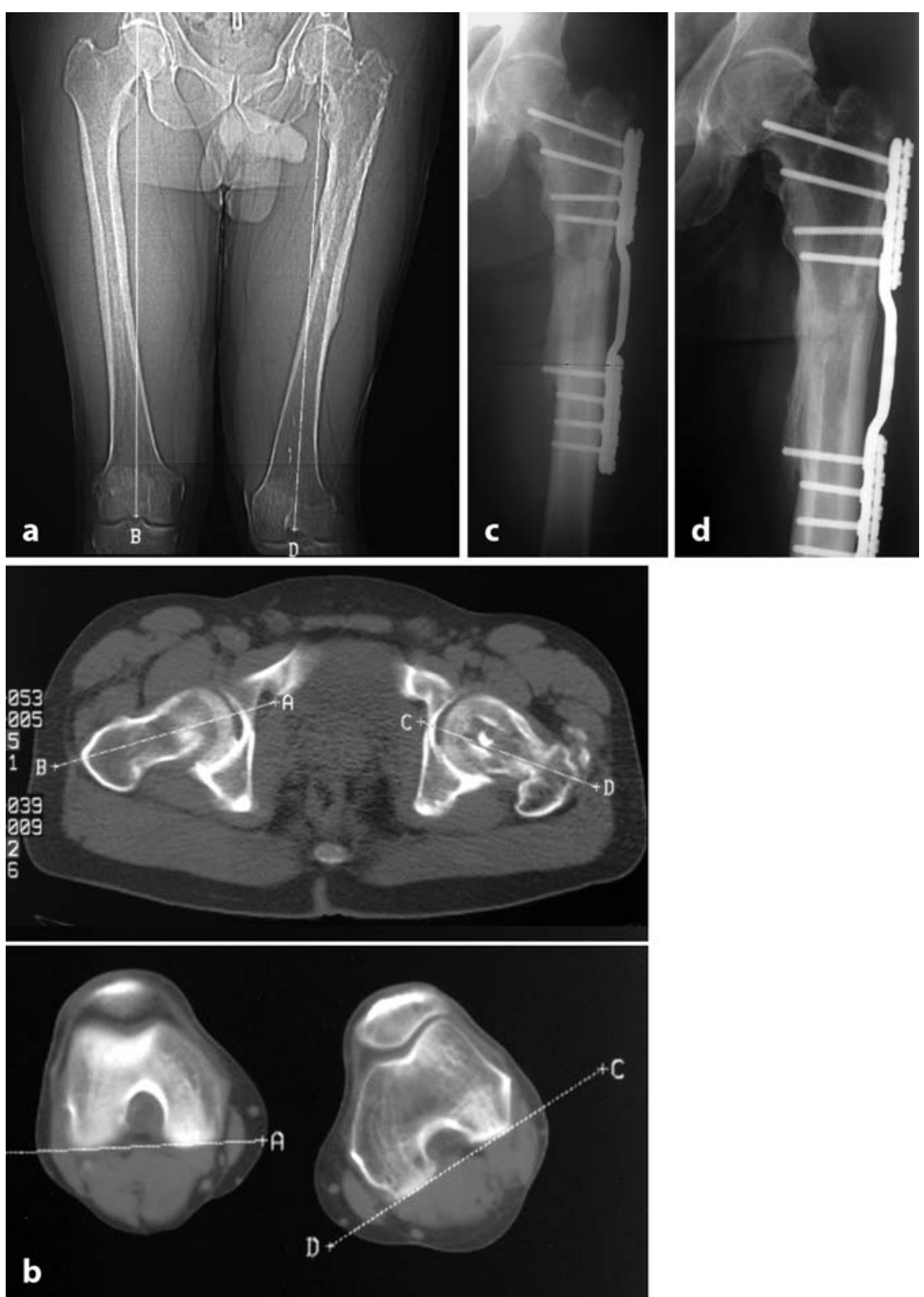

Abb. 4 a-d $\Delta$ 42-jähriger Patient, a in $40^{\circ}$ Innenrotationsfehlstellung verheilte Femurfraktur nach Winkelplattenosteosynthese, $\mathrm{b}$ CT-Untersuchung, hier zeigt sich die $40^{\circ}$-Innenrotationsfehlstellung, c Derotationsosteosynthese und Stabilisierung mit Druckplattenfixateur, $\mathrm{d}$ knöcherner Durchbau bei liegendem Druckplattenfixateur

gung mit Druckplattenfixateur und Spongiosaplastik.

Bei allen 3 Patienten kam es im weiteren Verlauf zu einer knöchernen Ausheilung mit Erreichen der Vollbelastung.

Bei 1 Korrekturosteotomie war eine Varusfehlstellung von $12^{\circ}$ verblieben.

\section{Diskussion}

Schaftfrakturen werden beim Erwachsenen bevorzugt durch Marknägel stabilisiert, wobei sich durch neue Verrie- gelungstechniken und Implantate die Indikationsstellung zur Frakturversorgung immer weiter in die proximale und distale Metaphyse verlagert hat [11]. Achsfehlstellungen ergeben sich v. a.im metaphysären Bereich. Hier weitet sich der Markraum trompetenförmig auf, sodass allein durch die Verriegelungsschrauben eine Achsabweichung nicht immer sicher zu vermeiden ist. Rotationsfehler mit einer Torsionsdifferenz von $>15^{\circ}$ wurden in der Literatur in bis $\mathrm{zu} 28 \%$ aller Femurmarknagelungen be- 

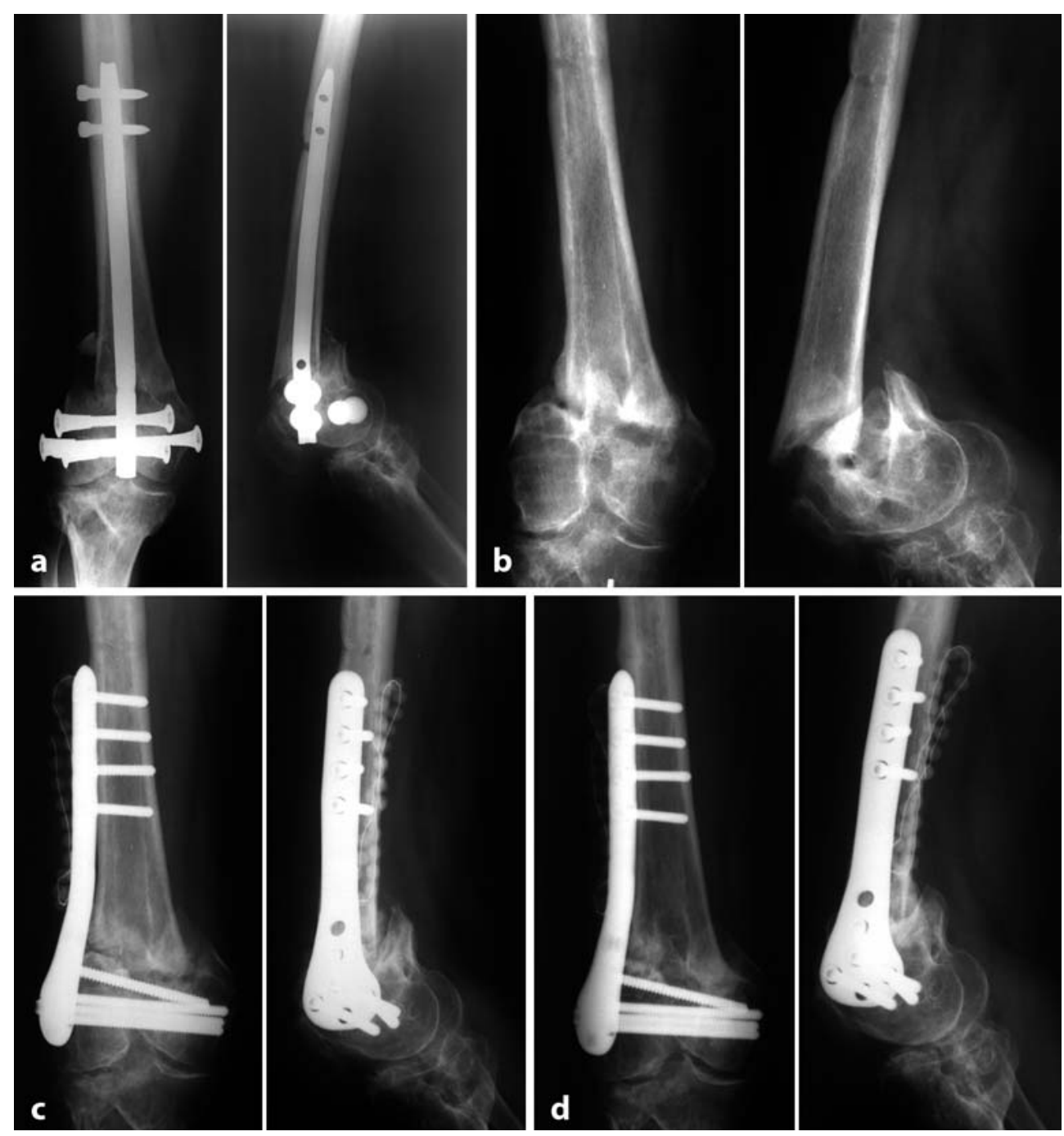

Abb. 5 a-d $>$ 49-jährige Patientin mit supra- und diakondylärer Oberschenkelfraktur, a mit retrogradem Marknagel stabilisiert, b nach Marknagelentfernung zeigt sich eine atrophe Pseudarthrose, c Stabilisierung mit Kondylen-TiFix, Anlagerung von autologer Spongiosa sowie Einlage von PMMA-Ketten $d$ knöcherner Durchbau der supraund diakondylären Oberschenkelfraktur dass das Volumen der DCS samt Spongiosaschraube etwa $38 \%$ größer ist. Die Auflagefläche von 4 winkelstabilen TiFix-Schrauben dagegen ist um etwa $70 \%$ größer als die einer DCS mit Spongiosaschraube. Dies bedeutet also für die Fixateur-interne-Platte eine größere knöcherne Abstützung bei geringerer Knochenschädigung durch großvolumige Implantate [6].

Bei den additiven Korrekturosteotomien (open wedge) kommt die Formbarkeit der Platte bei erhaltener Winkelstabilität besonders zum Tragen. Da die Osteotomien meist im metaphysären Bereich durchgeführt werden, kann durch Schränken der Platte der neuen knöchernen Geometrie nach additiver Osteotomie Rechnung getragen werden. Im Schaftbereich lassen sich durch den Druckplattenfixateur Torsionskorrekturen sicher ohne Korrekturverlust halten, insbesondere unter dem Gesichtspunkt, dass bei den älteren Marknagelsystemen das Bolzenspiel teilweise bis $\mathrm{zu} 25^{\circ}$ beträgt und hier einen sekundären Korrekturverlust zulässt [14].

Pseudarthrosen am Oberschenkel treten v. a. bei der Plattenosteosynthese am Femur auf (Abb. 3). Bei Durchführung der Plattenosteosynthese als elastische Osteosynthese hingegen wurden von Stürmer u. Schmidtmann [15] keine Pseudarthrosen am Femurschaft gesehen. Ursächlich hierfür werden die Weichteilschonung und das Freilassen von mindestens 2-4 Plattenlöchern über der Fraktur gesehen. Zusätzlich wird auf das Einpassen eines 3. Fragments verzichtet. Diese Voraussetzungen gelten auch bei der Pseudarthrosenversorgung. Ein Operationsverfahren mit Schonung von Periost, Markraum und Kortikalis wird den biologischen Gesetzmäßigkeiten der Frakturheilung wie Osteoregeneration, Osteovaskularisation und 
Fehlheilungen

Osteostabilisation am ehesten gerecht [12]. Weresh et al. [16] berichteten, dass 47\% der Pseudarthrosen nach Marknagelung, die durch erneute aufgebohrte Marknagelung therapiert wurden, nicht ohne einen weiteren Eingriff durchbauten. Insofern überzeugen die Fixateurinterne-Systeme mit ihren Ergebnissen (Abb. 5). Selbst bei Problempatienten (der Durchschnitt der Voroperationen lag bei 4,3) konnte in allen Fällen eine knöcherne Durchbauung erreicht werden. Bei den atrophen Pseudarthrosen, die 2/3 aller Pseudarthrosen ausmachten, war selbstverständlich immer eine Spongiosaplastik nötig (Abb. 5).

Der intraoperative Keimnachweis von $18,6 \%$ bestätigt uns in der regelhaften Einlage von PMMA-Ketten zur Infektprophylaxe. Dies schlägt sich auch in einer Infektionsrate von o\% nieder.

Die Problematik der atrophen Pseudarthrosen spiegelt sich in den Komplikationen wider. Hier traten 3 Plattenbrüche auf. Die Zytostatikatherapie eines Patienten mit chronisch-lymphatischer Leukämie erklärte den zögernden knöchernen Durchbau. Nach Plattenwechsel und erneuter Spongiosaplastik konnte letztendlich doch eine Ausheilung der Pseudarthrose erreicht werden. Der 2. Patient überlastete aufgrund von Uneinsichtigkeit bei Schmerzfreiheit das Implantat. Beim 3. Patient ist die Ursache des Implantatbruchs abgesehen von dem intraoperativ positiven Keimnachweis nicht weiter erklärlich.

Auch bei diesen beiden Patienten konnte nach Implantatwechsel und Spongiosaplastik ein knöcherner Durchbau erreicht werden.

\section{Fazit für die Praxis}

Die Fixateur-interne-Systeme erweisen sich insbesondere bei Problempatienten als hilfreich, um einen sicheren knöchernen Durchbau zu erreichen. Durch die Möglichkeit, die Platten zu biegen, wird man der anatomischen Form des Knochens bei erhaltener Winkelstabilität gerecht. Die frei wählbare Schraubenlage erlaubt eine hohe intraoperative Variabilität.

\section{Literatur}

1. Babin $S R$, Graf P,Vidal $P$, Sur $N$, Schvingt $E$ (1983) The risk non-union following closed-focus nailing and reaming. Results of 1059 interventions using the Kunstcher method. Int Orthop 7:133-143

2. Bostman 0, Varjonen L, Vainionpaa S et al. (1989) Incidence of local complications after intramedullary nailing and after plate fixations of femoral shaft fractures. J Trauma 29:639-645

3. Carr CR, Wingo CH (1973) Fractures of the femoral diaphysis: a retrospective study of the results and costs of treatment by intramedullary nailing and by traction and a spica cast. J Bone Joint Surg Am 55-A:690-700

4. Clatworthy MG, Clark DI, Gray DH, Hardy AE (1998) Reamed versus unreamed femoral nails: a randomised prospective trial.J Bone Joint Surg Br 80-B: 485-489

5. Eid AM, Deif Al (1980) Etiological factors in nonunion following Kuntscher intramedullary nailing of the femur. Arch Orthop Trauma Surg 96:213-220

6. Faschingbauer M, Wolter D Stütz A, Reimers N (1999) Distale Oberschenkelmehrfragmentfraktur. Ideale Indikation für ein winkelstabiles Implantat? Trauma Berufskrankh 1:337-343

7. Faschingbauer M, Stütz A, Wenzl M (2001) Fraktur- und Pseudarthrosenversorgung im Oberschenkelbereich durch Titan-Fixateur-interne-Systeme mit frei wählbarer Schraubenlage. Trauma Berufskrankh [Suppl 4] 3:434-438
8. Giannoudis PV, Furlong AJ, Macdonald DA, Smith RM (1997) Reamed against unreamed nailing of the femoral diaphysis; a retrospective study of healing time. Injury 28:15-18

9. Giannoudis PV, MacDonald DA, Matthews SJ, Schmith RM, Furlong AJ, De Boer P (2000) Nonunion of the femoral diaphysis.J Bone Joint Surg Br 82-B:5

10. Kamdar BA, Arden GP (1974) Intramedullary nailing for fractures of the femoral shaft. Injury 6:7-12

11. Kretteck C, Rudolf J, Schandelmaier P et al. (1996) Unreamed intramedullary nailing of femoral shaft fractures: operative technique and early clinical experience with the standard locking option. Injury 27:233-254

12. Schweiberer L, Baumgart R, Deiler St (1999) Die biologischen Bedingungen atropher und hypertropher Pseudarthrosen der Schaftknochen.Chirurg 70:1193-1201

13. Sennerich T, Sutter P, Ritter G, Zapf S (1992) Computertomographische Kontrolle des Antetorsionswinkels nach Oberschenkelschaftfrakturen des Erwachsenen. Unfallchirurg 95: 301-305

14. Strecker W, Suger G, Kinzl L (1996) Lokale Komplikationen der Marknagelung. Orthopäde 25: 274-291

15. Stürmer KM (1996) Die elastische Plattenosteosynthese, ihre Biomechanik, Indikation und Technik im Vergleich zur rigiden Osteosynthese. Unfallchirurg 99:816-829

16. Weresh MJ, Hakanson R, Stover MD, Sims SH, Kellam JF, Bosse MJ (2000) Failure of exchange reamed intramedullary nails for ununited femoral shaft fractures J Orthop Trauma 14: 335-338

17. Wickstrom J, Corban M, Vise G (1968) Complications following intramedullary fixation of 324 femoral fractures. Clin Orthop 60: 103-113

18. Wolinsky PR, McCarty E, Shyr Y, Johnson K (1999) Reamed intramedullary nailing of the femur:551 cases. J Trauma 46:392-399

19. Wolter $D$ (1991) Der Plattenfixateur interne für lange Röhrenknochen. In:Wolter D, Zimmer W (Hrsg) Die Plattenosteosynthese und ihre Konkurrenzverfahren. Springer, Berlin Heidelberg New York

\section{Achskorrekturen und Pseudarthrosenbehandlung nach Unterschenkelfrakturen mit Marknagel}

Autor hat kein Manuskript eingereicht 